\title{
For which diseases do broader value elements matter most? An evaluation across 20 ICER evidence reports
}

\author{
Jason Shafrin, PhD; Syvart Dennen, MPhil; Priti Pednekar, PhD; Kelly Birch, MPH; Menaka Bhor, PhD; Julie Kanter, MD; \\ and Peter Neumann, ScD
}

\section{What is already known about this subject}

- Policymakers, payers, and other stakeholders in the health care sector are increasingly using cost-effectiveness analysis (CEA) to quantify the value of new interventions.

- Historically, CEAs have used a health system perspective in measuring value, without fully accounting for potential societal benefits and novel value elements.

\section{What this study adds}

- The current study reviewed and analyzed evidence reports from the U.S.-based Institute for Clinical and Economic Review to identify the degree to which broader value elements (e.g., caregiver burden, productivity loss) may be relevant for evaluation of treatments for various diseases.

- The results aim to provide guidance to stakeholders regarding which disease areas should be prioritized for future research to quantify these broader elements for inclusion in value assessments.

\section{Author affiliations}

Jason Shafrin, PhD; Syvart Dennen, MPhil; Priti Pednekar, PhD; and Kelly Birch, MPH, PRECISIONheor, Los Angeles, CA. Menaka Bhor, PhD, Exact Sciences Corporation, Madison, Wl; Julie Kanter, MD, University of Alabama at Birmingham; and Peter Neumann, ScD, Center for the Evaluation of Value and Risk in Health, Institute for Clinical Research and Health Policy Studies, Tufts Medical Center, Boston, MA.

\section{AUTHOR CORRESPONDENCE:}

Syvart Dennen, 310.984.7729;

Syvart.Dennen@precisionvh.com

J Manag Care Spec Pharm. 2021;27(5):650-59

Copyright $\odot 2021$, Academy of Managed Care Pharmacy. All rights reserved.

status, treatment cost, estimated treatment cost-effectiveness, and ICER committee voting results for contextual considerations and additional benefits/disadvantages.

RESULTS: The most commonly cited broader value element category in the ICER evidence reports was household and leisure (e.g., absenteeism from normal activities and caregiver burden). More value elements were cited for inherited retinal disease (19 elements) and sickle cell disease (18 elements) than for other diseases. Cardiovascular disease and diabetes had the fewest number of value elements cited (7 elements). Rare diseases were more likely to have broader value elements cited compared with nonrare 
diseases ( 15.9 vs. $11.5, P<0.001)$. Treatments with higher (i.e., less favorable) incremental cost-effectiveness ratios were more likely to have a greater number of broader value elements cited $(\rho=0.625$, $P<0.001)$.

CONCLUSIONS: The presence of broader value elements varied across diseases, with less cost-effective treatments more likely to have a higher number of relevant broader value elements. Inclusion of all relevant value elements in value assessments will more appropriately incentivize innovation and improve allocation of research funding.

In recent years, policymakers, payers, and other stakeholders in the health care sector have increasingly used cost-effectiveness analysis (CEA) to quantify and communicate the value of new interventions. ${ }^{1}$ Major value framework developers in the United States such as the Institute for Clinical and Economic Review (ICER), the American College of Cardiology, the American Heart Association, and the American Society for Clinical Oncology use CEA in their assessments of health care technologies. In traditional CEA, the direct costs and the individual health benefits and risks of an intervention are compared with at least 1 alternative, and the intervention's value is estimated as a ratio of incremental cost to incremental net impact on health. Historically, the majority of CEAs have used a health system perspective without fully accounting for societal and broader benefits and costs due to an intervention, with just $29 \%$ of studies through 2005 taking a societal perspective. ${ }^{2}$ Furthermore, CEAs conducted from a payer or health system perspective do not typically account for patient-reported outcomes of specific treatments or broader outcomes, such as the ability to return to work or reduction in caregiver burden. ${ }^{3}$

Several recent studies and consensus panels agree that wider individual and societal benefits should be accounted for in CEAs and have enumerated broader elements of value that the traditional health care sector perspective may neglect. In 2016, the Second Panel on Cost-Effectiveness in Health and Medicine (the "Second Panel" hereafter) provided recommendations for improving the quality of CEA studies. One of the Second Panel's recommendations was the inclusion of a societal perspective in addition to the traditional health care sector perspective, with the addition of elements including, but not limited to, time costs for patients and caregivers, transportation costs, effects on future consumption and productivity, and effects on educational achievement. The Second Panel provides an impact inventory that encourages researchers to explicitly consider the broader health and nonhealth effects of the intervention.
Additionally, the International Society for Pharmacoeconomics and Outcomes Research (ISPOR) released a Special Task Force report in 2018 advocating for the exploration of augmenting CEAs with novel value elements. ISPOR organizes elements of value used in CEA into core elements (quality-adjusted life-years [QALYs] and net costs), commonly but inconsistently used elements (productivity and adherence-improving factors), and novel elements that may be considered when conducting CEA from a societal perspective. These novel value elements include insurance value, disease severity, the value of hope, real option value, health equity, scientific spillovers, reduction in uncertainty of outcomes, and fear of contagion. ${ }^{3}$ Insurance value is the value that healthy individuals place on protection against the risk of financial and health loss should they become ill in the future. ${ }^{3,4}$ When probability of survival is poor, the value of hope is the incremental value placed on the tail of the survival curve above and beyond differences in mean expected life extension relative to a comparator. ${ }^{3,5}$ Real option value is the added value when treatment that extends life creates opportunities for the patient to benefit from other future health technology advances. ${ }^{3}$

Furthermore, a systematic review conducted by Drost et al. (2017) found that approximately $69 \%$ of CEA studies meeting inclusion criteria incorporated 1 or more elements of a societal perspective. Productivity costs were the most commonly included element (67\% of studies), with few studies addressing elements related to caregivers (5\%) or the educational sector (2\%). ${ }^{1}$

Although there is ongoing debate about the most appropriate methods for including broader value elements in value assessment, it remains unclear whether the inclusion of these value elements is likely to affect the quantitative estimates of treatment value. A recent study reviewed published ICER reports and analyzed the relationship among interventions' incremental cost-effectiveness, the votes by ICER appraisal committee members on each category of potential other benefits and contextual considerations, and the overall votes of appraisal committee members on longterm value for money. The study found that interventions with lower (i.e., more favorable) cost-effectiveness ratios were more likely to receive high or intermediate numbers of votes on long-term value for money. In the "other benefits and disadvantages" category, ICER's appraisal committee awarded the greatest number of votes to the element of having a novel mechanism of action, whereas, in the "contextual considerations" category, treating conditions with a severe impact on length and quality of life received the most votes. ${ }^{6}$ However, the study did not examine the relationships between the number of broader value elements and incremental cost-effectiveness. 
In summary, multiple studies, panels, and task forces have explored the current use of broader value elements in CEA and recommended their incorporation into future value assessments. Further research is needed to identify and measure these novel value elements across disease areas. Our study aims to provide guidance to stakeholders regarding which disease areas should be prioritized for future research into quantifying these broader elements.

\section{Methods}

This study used a 5-step approach to identify the degree to which broader value elements are relevant for evaluation of treatments for various diseases. First, we conducted a targeted review of the academic literature and the value assessment recommendations of leading U.S. institutions to identify broader value elements. Second, we selected a range of CEAs performed by ICER under a consistent value assessment framework. Third, we examined the selected draft or final ICER evidence reports to determine which diseases had a greater number of broader value elements discussed as important to the disease or included in ICER's CEA framework. Fourth, we evaluated the relationships between the number of broader value elements in each report, disease characteristics, drug costs, and incremental cost-effectiveness. Finally, for each final evidence report, ICER held a policy roundtable discussion with various stakeholders and asked panelists to vote on the relevance of contextual considerations. In the fifth and final step, we analyzed these voting patterns to explore relationships of voting results with broader value elements, drug costs, and incremental cost-effectiveness.

Our methodology for selecting ICER reports and identifying broader elements of value to be included in the analysis is described in the supplementary material (see Additional Methodology, available in the online article). The following sections describe our qualitative and quantitative analyses.

\section{IDENTIFYING THE NUMBER OF RELEVANT BROADER VALUE ELEMENTS ACROSS DISEASES}

ICER reports from the defined study period (July 2017January 2020) were reviewed for text discussing broader elements of value. Two coders (PP and $\mathrm{KB}$ ) independently assigned text segments to the set of predefined value element categories using MAXQDA software (Version 12.3.5, VERBI). A set of predefined search terms was used to find relevant text, followed by a manual review of the full report. A third individual (SD) reviewed the coded text segments for accuracy and served as a tiebreaker in case of conflict. We coded text segments only if they discussed a value element as relevant to the disease or listed the element as a component of ICER's CEA approach for the specific disease. Each value element was counted only once regardless of the number of times it was discussed within a report.

\section{ASSOCIATION AMONG THE NUMBER OF BROADER VALUE ELEMENTS AND DRUG COSTS, COST-EFFECTIVENESS RATIOS, AND DISEASE CHARACTERISTICS}

If pharmaceutical firms use a value-based approach to pricing that leads to high-cost treatments being targeted to diseases with a wide range of impacts on individuals and society, there may be a positive correlation between drug costs and the number of broader value elements. We report the Pearson correlation coefficient $(\rho)$ between the number of reported value elements and both lifetime discounted drug cost and treatments' incremental cost-effectiveness ratios. Results should be viewed with caution due to the small sample size.

Broader value elements may be more commonly discussed for some diseases than others. We therefore evaluated the number of value elements by disease characteristic (i.e., rare vs. nonrare disease, oncology vs. nononcology). Rare diseases were classified twice, using definitions from ICER and the U.S. Food and Drug Administration (FDA). ICER defines ultrarare disease as having a prevalence of less than 10,000 individuals in the United States, ${ }^{7}$ whereas the FDA Office of Orphan Products Development rare disease definition is a prevalence of less than 200,000 persons in the United States. ${ }^{8}$ The statistical significance of differences in number of value elements for rare versus nonrare diseases under the FDA definition was evaluated through Welch's t-test, which has been shown to provide a type I error rate close to the nominally specified rate in small samples, albeit at the cost of reduced power. ${ }^{9}$ Due to small sample size, the results of these tests should still be interpreted with caution.

\section{VOTING PATTERNS}

In ICER deliberation meetings, appraisal committee members vote on whether an intervention has "potential other benefits or disadvantages" or impacts "contextual considerations." These categories represent benefits or disadvantages by the intervention to the patient, caregivers, or the public that may not have been captured in the conventional analyses. Hence, the voting patterns indicate whether the appraisal committee feels that these value elements should be considered alongside the cost-effectiveness results while making pricing decisions.

We evaluated ICER committee voting patterns for contextual considerations and additional benefits/ 


\section{TABLE 1}

Disease Areas/Interventions Investigated in ICER Evidence Reports Reviewed in the Study

\begin{tabular}{l|c}
\hline \multicolumn{1}{c|}{ Condition/Intervention } & $\begin{array}{c}\text { Year of Final } \\
\text { Report }\end{array}$ \\
\hline Diabetes & 2020 \\
\hline Acute migraine & 2020 \\
\hline Sickle cell disease & 2020 \\
\hline Cardiovascular disease & 2019 \\
\hline Depression & 2019 \\
\hline Duchenne muscular dystrophy & 2019 \\
\hline Multiple sclerosis & 2019 \\
\hline Peanut allergy & 2019 \\
\hline Spinal muscular atrophy & 2019 \\
\hline Amyloidosis & 2018 \\
\hline Asthma & 2018 \\
\hline CAR T-cell therapy for ALL/DLBCL & 2018 \\
\hline Endometriosis & 2018 \\
\hline Hemophilia & 2018 \\
\hline Hereditary angioedema & 2018 \\
\hline Episodic or chronic migraine & 2018 \\
\hline Opioid use disorder & 2018 \\
\hline Prostate cancer & 2018 \\
\hline Psoriasis & 2018 \\
\hline Ecoretigene neparvovec for inherited retinal disease & 2018 \\
\hline
\end{tabular}

disadvantages across disease areas. Weighted voting results were calculated as the sum of the proportions of votes in favor of contextual considerations and additional benefits/ disadvantages and were summarized by disease area and intervention. We examined the relationship between the weighted voting results, the counts of value of elements reported, lifetime discounted drug costs, and incremental cost-effectiveness ratios (cost/QALY). The validity of this approach hinges on the assumption that the share of committee members who voted for a given contextual consideration or additional benefit or disadvantage is positively correlated with a value element's relative importance. For instance, if caregiver burden for a given disease is high and likely to be affected by the intervention in question, one would expect most members of the ICER policy roundtable to vote that this element is relevant.
To explore the relationship between voting patterns and the count of elements by disease, we calculated Pearson's correlation between these measures across ICER reviews. We similarly report the correlation between voting results and the lifetime discounted drug cost and incremental cost per QALY. Due to small sample size, the correlations and reported $\mathrm{P}$ values should be viewed with caution.

\section{Results}

\section{ENUMERATION OF BROADER VALUE ELEMENTS}

Our literature search identified 32 disease-specific and 6 intervention-specific broader value elements. The 32 disease-specific value elements were divided into 9 categories: productivity, education, criminal justice system, household and leisure, health equity, social services/public spending, risk preference, scientific spillovers, and other. These category definitions were based on the combination of those reported in the ICER value frameworks, the Second Panel, ISPOR special task force recommendations, and academic literature..$^{1,3,10-14}$ The full list of individual broader value elements is listed in Supplementary Table 1 (available in online article).

\section{BROADER VALUE ELEMENTS ACROSS ICER EVIDENCE REPORTS}

A total of 20 ICER evidence reports were reviewed to capture broader value elements reported across disease areas (Table 1). These reports evaluated 49 treatments across 21 diseases. Diseases represented included amyloidosis, asthma, cardiovascular disease, acute lymphocytic leukemia, diffuse large B-cell lymphoma, depression, diabetes, Duchenne muscular dystrophy, endometriosis, hemophilia, hereditary angioedema, inherited renal disease, chronic migraine, acute migraine, multiple sclerosis, opioid use disorder, peanut allergy, prostate cancer, psoriasis, sickle cell disease, and spinal muscular atrophy. Of these diseases, 2 were ultrarare per ICER's definition, 8 were rare based on the FDA definition, and 2 were oncology-related (Supplementary Table 2, available in online article).

All evidence reports included the broader value element categories of productivity, household and leisure, and health equity (Supplementary Figure 1 and Supplementary Table 3, available in online article). Education-related value elements were cited in 12 evidence reports, elements related to social services and risk preference in 2 evidence reports, and elements related to the criminal justice system in 1 report. Scientific spillovers were not mentioned, except through the "novel mechanism of action" element in the "intervention-specific" category. 


\section{FIGURE 1 Count of Broader Value Elements Cited Across 20 ICER Reports, by Category}

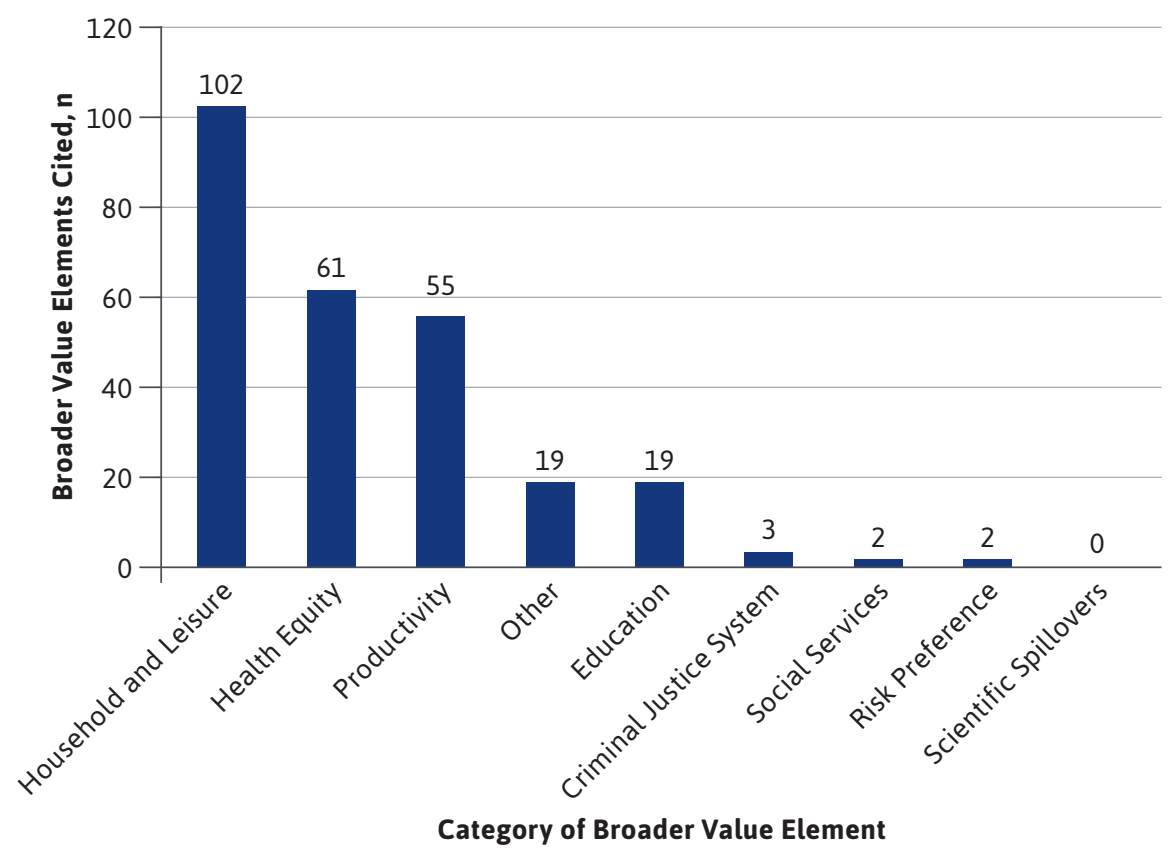

ICER = Institute for Clinical and Economic Review.

Elements from the household and leisure category were cited 102 times in 263 total citations, making it the most common category (Figure 1 and Supplementary Table 3). The second most frequently discussed value element category was health equity, with 61 of the 263 citations. This category includes elements such as high lifetime burden of disease and health disparities. Productivity, which captures elements such as work absenteeism and lost income, was ranked third with 55 citations. Elements related to education were discussed 19 times. Elements in the criminal justice system, social services, and risk preference categories were rarely mentioned, with $\leq 3$ citations overall. ICER did not discuss scientific spillovers in any of the included evidence reports.
In 19 of the 20 reports, ICER discussed some value elements that did not fit clearly into any of our predefined categories. These included elements such as stigma surrounding the disease and the failure of QALYs to adequately capture disease effects. ICER and the Second Panel both include an "other" category for elements outside the predefined categories, and we, therefore, included this category in the present study. The "other" category was treated as a single element and contributed only once to the total count of elements for each of the 19 reports where it was included. In practice, inclusion of the "other" category did not affect the rank ordering of disease by count of value elements.

\section{BROADER VALUE ELEMENTS ACROSS DISEASE AREAS}

Inherited retinal disease (with 19 value elements) and sickle cell disease (with 18) had the greatest number of broader value elements cited (Figure 2). Amyloidosis and Duchenne muscular dystrophy (with 17 elements) had the third greatest number of cited elements, and depression ranked fourth (with 16 elements). Cardiovascular disease and diabetes had the fewest value elements (7 elements each), followed by prostate cancer (10 elements) and peanut allergy, asthma, endometriosis, and acute migraine (11 elements).

The number of elements included per category varied across the disease areas (Figure 2 and Supplementary Table 3 ). The inherited retinal disease report had the most value elements in the household and leisure category (9 elements), whereas cardiovascular disease and opioid use disorder had the fewest (1 element each). Inherited retinal disease, amyloidosis, and spinal muscular atrophy had the greatest number of health equity elements (5 elements each). Productivity elements were most often discussed for depression, acute migraine, and hemophilia (4 elements each). Criminal justice system-related elements were captured only for opioid use disorder. Risk preferences, which include insurance value, real option value, and the value of hope, were discussed only in the 2 reports related to oncology: CAR T-cell therapies for lymphomas, leukemia, and prostate cancer. Value elements related to social services were discussed only in the reports for opioid use disorder and inherited retinal disease.

\section{RELATIONSHIP OF BROADER VALUE ELEMENTS WITH DRUG COSTS AND COST-EFFECTIVENESS RATIOS}

Reports on less cost-effective drugs discussed more value elements on 


\section{FIGURE 2 Disease-Specific Broader Value Elements Across Diseases}

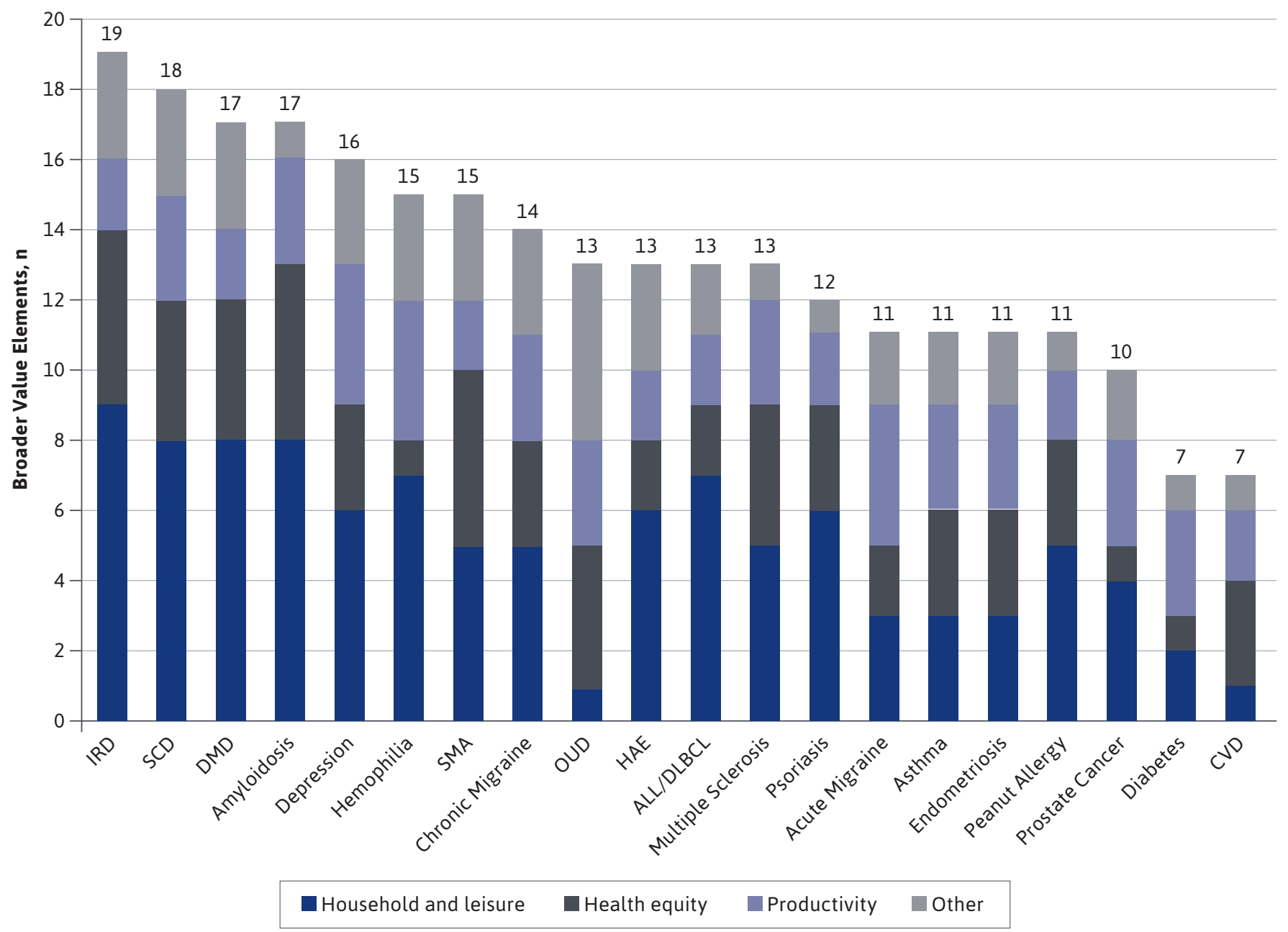

$A L L=$ acute lymphocytic leukemia; $C V D=$ cardiovascular disease; $D L B C L=$ diffuse large $B$-cell lymphoma; $D M D=$ Duchenne muscular dystrophy; IRD =inherited retinal disease; $H A E$ = hereditary angioedema; $O U D=$ opioid use disorder; $S C D=$ sickle cell disease; $S M A=$ spinal muscular atrophy.

average. The Pearson correlation between incremental costeffectiveness ratios and the number of value elements was $0.356(\mathrm{P}=0.021)$. After a natural $\log$ transformation of the cost-effectiveness ratio to better account for the right skew of the data, the correlation increased to $0.625(\mathrm{P}<0.001$, Figure 3). The 10 least cost-effective treatments averaged 17.0 value elements, whereas the 10 most cost-effective drugs averaged 9.6 value elements (Supplementary Table 4, available in online article). Cost-effectiveness ratios for treatments for Duchenne muscular dystrophy, hemophilia, opioid use disorder, and acute migraine were not reported and, thus, were excluded from the analysis.

The 10 highest-priced drugs had an average of 15.0 value elements cited, whereas the 10 lowest-priced drugs had an average count of 11.1 value elements $(P=0.006$; Supplementary Table 4). The lifetime discounted costs of treatments for hereditary angioedema, Duchenne muscular dystrophy, opioid use disorder, endometriosis, amyloidosis, chronic migraine, and psoriasis were not reported, and, thus, these treatments were not included in this analysis. 


\section{FIGURE 3 Scatterplot of ICER Reports by the Number of Broader Value Elements and Cost-Effectiveness}

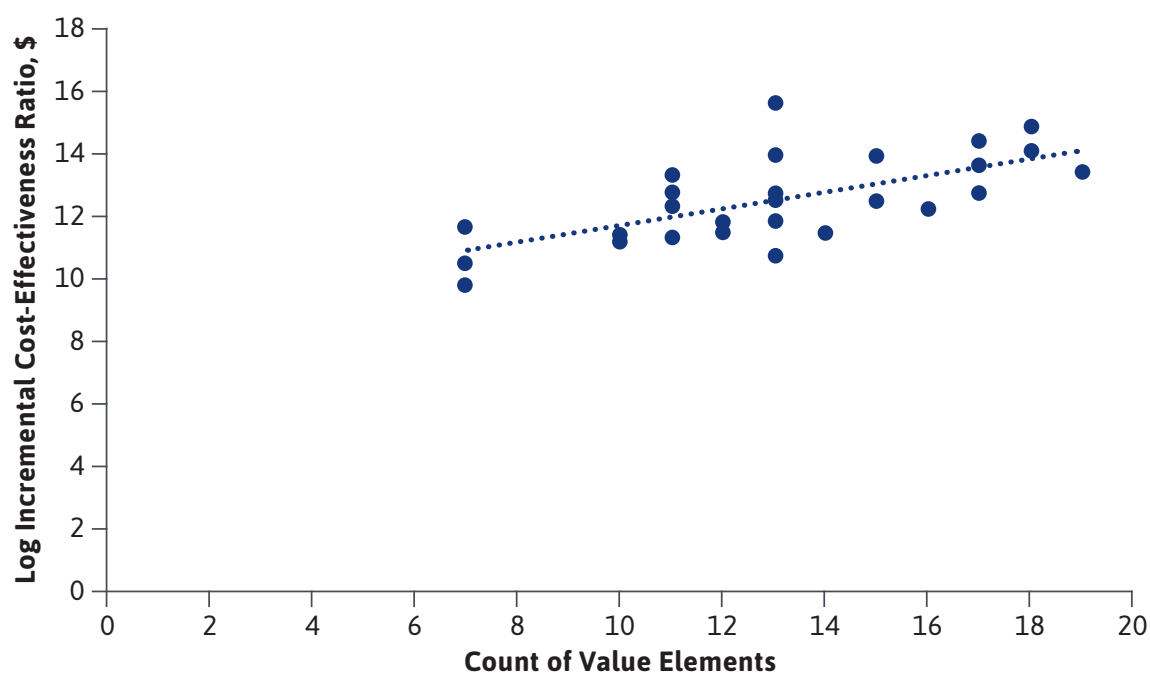

Note: Y-axis represents natural log of cost-effectiveness ratio. Cost-effectiveness ratios for treatments for Duchenne muscular dystrophy, hemophilia, opioid use disorder, and acute migraine were not provided in the ICER reports, and these unknown cost-effectiveness ratios were excluded from the analysis. ICER = Institute for Clinical and Economic Review.

\section{BROADER VALUE ELEMENTS FOR RARE DISEASES AND ONCOLOGY}

Reports on rare diseases included discussion of a greater average number of broader value elements than nonrare-disease reports. According to the FDA definition, 8 of the 21 conditions evaluated were rare diseases (Supplementary Table 2). Following this definition, rare diseases had $38 \%$ more elements of value (15.9 vs. $11.5, \quad \mathrm{P}<0.001)$ compared with nonrare diseases. Only amyloidosis and Duchenne muscular dystrophy were classified as ultrarare under ICER's definition (Supplementary Table 2). These diseases had a 34\% greater average number of value elements compared with nonrare diseases (17.0 vs. 12.7). Reports covering cancer (2 of 20 reports) discussed 11.5 average elements and were the only reports to discuss risk preference elements. Due to small sample size, statistical testing results are not provided for the latter 2 comparisons.

\section{ICER VOTING RESULTS}

ICER voting results varied widely across disease areas. Whereas our evaluation of value elements occurred at the disease level, voting results in this study are reported by intervention. Inotersen and patisiran for amyloidosis had the highest weighted vote totals for contextual considerations and additional benefits/ disadvantages, followed by voretigene neparvovec for inherited retinal disease and calcitonin gene-related peptide inhibitors for chronic migraine (Figure 4). Diabetes and prostate cancer had the lowest weighted vote totals. Voting results exhibited some differences among treatments within the same disease. For instance, sublocade had higher weighted vote totals than probuphine (5.7 vs. 4.8 ), despite the fact that both interventions are indicated for the treatment of patients with opioid use disorder. This finding was driven by differences in votes of ICER panel members on whether the intervention reduced complexity and whether it was a novel treatment where others had failed. Voting results for contextual considerations and additional benefits/disadvantages for sickle cell disease and acute migraine were not available in the draft evidence reports.

The weighted voting results were highly correlated with the number of broader value elements cited for a given treatment, with $\rho=0.597$ $(P<0.001)$ (Supplementary Figure 2). Drugs with higher lifetime discounted costs tended to have higher weighted vote totals, with $\rho=0.399$; however, the relationship was not statistically significant $(P=0.073)$. Incremental cost per QALY was positively but nonsignificantly correlated with weighted vote totals, with $\rho=0.128 \quad(P=0.484)$. A natural $\log$ transformation of incremental cost per QALY showed a stronger association, with $\rho=0.357$ $(P=0.045)$. Interventions with missing information on votes, lifetime discounted costs, and cost-effectiveness ratios were not included.

\section{Discussion}

This study found evidence of significant heterogeneity in the number of broader value elements cited in ICER evidence reports across diseases. Inherited retinal disease and sickle cell disease had the greatest number of broader value elements discussed by ICER reports, whereas cardiovascular disease and diabetes had the fewest. Further, though the sample size was small, diseases classified as rare had numerically higher counts of value elements than nonrare diseases. This heterogeneity may be attributable to the fact that not all elements are 


\section{FIGURE 4 Weighted Voting Results, by Disease and Intervention}

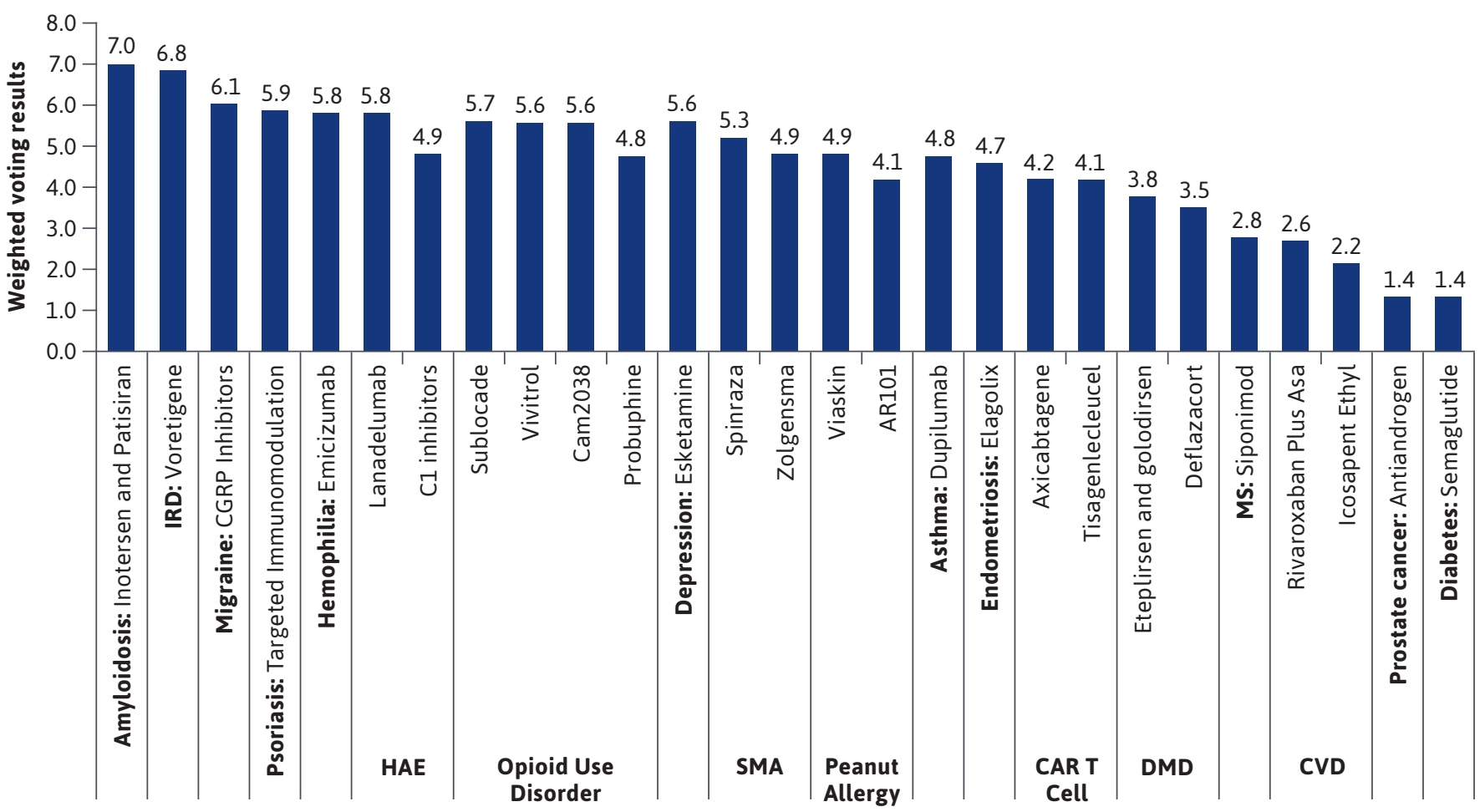

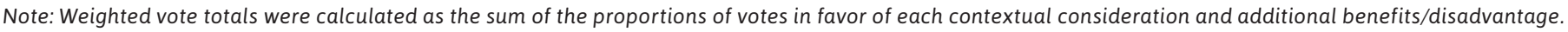
CAR T cell=chimeric receptor antigen T cell; CGRP=calcitonin gene-related peptide; CVD=cardiovascular disease; DMD=Duchenne muscular dystrophy; $I R D=$ inherited retinal disease; $H A E=$ hereditary angioedema; OUD=opioid use disorder; SCD =sickle cell disease; $S M A=$ spinal muscular atrophy.

relevant to all disease areas. For example, elements related to the criminal justice system are important in understanding the impacts of opioid use disorder but are unlikely to be relevant for a disease like prostate cancer. Value elements related to risk preferences and scientific spillovers may be highly important for some diseases but were rarely discussed by ICER.

If value assessment ignores a large number of value elements, treatments that have wide-ranging impacts may be undervalued and receive inappropriately high incremental cost-effectiveness ratios. We found an association between the number of value elements and the incremental costeffectiveness ratios, providing some evidence that this may be the case. In addition, we found that rare-disease reports cited more elements on average than nonrare-disease reports, which may put rare diseases at greater risk of undervaluation. Furthermore, when evaluating treatments across different disease areas, excluding relevant value elements means that interventions are not measured in a way that allows for an accurate comparison of their full costs and benefits.

Failing to incorporate broader value elements has 2 primary health policy implications. First, if prices are linked to value, payers may be underpaying for certain treatments because value may be too narrowly defined. If treatments for diseases that impact many value elements are undervalued, incentives for innovation in these areas will be reduced. For example, failure to capture important elements such as unmet need, insurance value, and health equity can stifle innovation in treatments for rare diseases..$^{15}$ Second, government research dollars may not be channeled to funding for treatments with the highest disease burden. Cystic fibrosis, for example, has received 3.5 times greater funding per capita from the National Institutes of Health than sickle cell disease. ${ }^{16}$ Whether 1 of these diseases should in fact receive relatively more or less funding cannot be meaningfully discussed without a full accounting of the elements of value relevant for each condition. 
Policymakers and other stakeholders should also consider additional funding to inform methods for better quantifying novel value elements and mechanisms for incorporating broader value elements into decision making. Researchers have been working to improve identification and estimation of value elements for years, and progress continues to be made, ${ }^{17}$ such as recent research that has identified novel value elements and described methodology for quantifying them. ${ }^{3}$ More methodological research, however, is needed. Additional health policy research should examine robust approaches for incorporating broader value elements into decision making. One approach, for instance, would be to simply augment standard traditional CEA with these broader value elements. Another possible approach would be to include broader value elements as criteria in a multicriteria decision analysis (MCDA). MCDA provides a framework for expert panels to weight the relevant value elements more rigorously than traditional CEA. Open-source economic models developed by the Innovation and Value Initiative focusing on rheumatoid arthritis model and non-small cell lung cancer model have incorporated an MCDA framework. ${ }^{18}$

\section{LIMITATIONS}

This study has several limitations. First, for consistency, the study included only ICER evidence reports; no other sources of health technology assessments were included. We recommend that future research consider replicating this exercise using value assessments from institutions outside the United States, such as value assessments from the National Institute for Health and Care Excellence.

Second, the number of ICER reports covered was modest $(n=20)$. Although the research team considered examining a larger number of diseases, the use of recent ICER reports ensured that the evaluations were both recent and used a homogeneous, structured approach to value assessment in the United States.

Third, although our search for broader value elements employed well-cited publications in this area, there are likely value elements that were excluded. For example, other research has focused on broader value elements for specific types of interventions, such as vaccines, ${ }^{19,20}$ and these studies' value elements were not explicitly included in our study.

Fourth, the value elements were captured using the ICER report text, which could be subject to bias if some reports provide more comprehensive review of value elements than others. Despite this limitation, using a single source of information across diseases (i.e., ICER reports) is likely to minimize the magnitude of this bias.

Fifth, and most important, this study measures the frequency with which value elements appear across diseases but does not quantify the magnitude of the value element within a given disease nor how it should be valued. The study used ICER panel voting results to examine committee members' judgments about which broader value elements are important to consider when valuing a given intervention. Although this approach is valid if voting proportions for a given value element are correlated with the magnitude of that value element, the evidence should be interpreted as suggestive only.

\section{Conclusions}

The number of broader elements of value cited in ICER reports differed widely across diseases, with inherited retinal disease and sickle cell disease having the highest count of broader value elements cited and cardiovascular disease and diabetes having the lowest. Rare diseases had more value elements cited compared with nonrare diseases. There was a positive relationship between a treatment's incremental cost-effectiveness ratio and the number of broader elements cited in ICER reports. Inclusion of all relevant value elements in value assessments will more appropriately incentivize innovation and improve allocation of research funding. Future research should aim to better quantify these value elements and could consider other techniques, such as MCDA, to prioritize the relevance of these value elements across diseases.

\section{DISCLOSURES}

This study was sponsored by Novartis Pharmaceutical Corporation. At the time of this study, Shafrin was employed by PRECISIONheor, a consultancy to the life sciences industry that received financial support from Novartis to conduct this study. Dennen, Pednekar, and Birch are employed by PRECISIONheor. Bhor was an employee of Novartis Pharmaceutical Corporation at the time this research was conducted and manuscript was developed and reports grants from Novartis, unrelated to this work. Kanter has served on scientific advisory boards and steering committees for and reports receiving consulting fees from Novartis Pharmaceutical Corporation and is a site principal investigator on studies funded by Novartis Pharmaceutical Corporation. Kantar also reports support from Sickle Cell Disease Association of America Inc. and National Heart, Lung, and Blood Institute, unrelated to this work. Neumann reports advisory boards or consulting fees from Novartis Pharmaceutical Corporation and PRECISIONheor, as well as advisory boards or consulting fees unrelated to this study from AbbVie, Amgen, Avexis, Bayer, Congressional Budget Office, Janssen, Merck, Novartis, Novo Nordisk, Precision Health Economics, Veritech, Vertex; funding from The CEA Registry Sponsors by various pharmaceutical and medical device companies; and grants from Amgen, Lundbeck, Bill and Melinda Gates Foundation, National Pharmaceutical Council, Alzheimer's Association, and the National Institutes for Health. 


\section{REFERENCES}

1. Drost R, van der Putten IM, Ruwaard D, Evers S, Paulus ATG. Conceptualizations of the societal perspective within economic evaluations: a systematic review. Int J Technol Assess Health Care. 2017;33(2):251-60.

2. Neumann P. Costing and perspective in published cost-effectiveness analysis. Medical Care. 2009;47(7 Suppl 1):S28-32.

3. Lakdawalla D, Doshi J, Garrison L, Jr., Phelps C, Basu A, Danzon P. Defining elements of value in health care-a health economics approach: an ISPOR Special Task Force report. Value Health. 2018;21(2):131-39.

4. Lakdawalla D, Malani A, Reif J. The insurance value of medical innovation. J Public Econ. 2017;145:94-102.

5. Garrison L, Towse A. Value-based pricing and reimbursement in personalised healthcare: introduction to the basic health economics. J Pers Med. 2017;7(3):10.

6. Trenaman L, Pearson S, Hoch J. How are incremental cost-effectiveness, contextual considerations, and other benefits viewed in health technology assessment recommendations in the United States? Value Health. 2020;23(5):576-84.

7. Institute for Clinical and Economic Review. Modifications to the ICER value assessment framework for treatments for ultra-rare diseases 2017. November 2017. Accessed March 4, 2021. https:// icer.org/wp-content/uploads/2020/10/ ICER-Adaptations-of-Value-Frameworkfor-Rare-Diseases.pdf
8. U.S. Food and Drug Administration. Developing products for rare diseases \& conditions. December 20, 2018. Accessed March 4, 2021. https://www.fda.gov/ industry/developing-products-rare-diseases-conditions

9. De Winter, JC. Using the Student's t-test with extremely small sample sizes. Practical Assess Res Eval. 2013;18(1):10.

10. Institute for Clinical and Economic Review. Overview of the ICER value assessment framework and update for 2017-2019. 2019. Accessed March 4, 2021. https://icer.org/ wp-content/uploads/2020/10/ ICER-value-assessment-frameworkUpdated-050818.pdf

11. Institute for Clinical and Economic Review. 2020 value assessment framework: proposed changes. August 21, 2019. Accessed March 4, 2021. https://icer. org/wp-content/uploads/2020/10/ ICER 2020 VAF Proposals 082119-1.pdf

12. Sanders G, Neumann P, Basu A, et al. Recommendations for conduct, methodological practices, and reporting of cost-effectiveness analyses: second panel on cost-effectiveness in health and medicine. JAMA. 2016;316:1093-103.

13. Willke R, Neumann P, Garrison L, Jr., Ramsey S. Review of recent U.S. value frameworks-a health economics approach: an ISPOR Special Task Force Report [6]. Value Health. 2018;21(2):155-60.
14. Garrison L Jr., Pauly M, Willke R, Neumann P. An overview of value, perspective, and decision context-a health economics approach: an ISPOR Special Task Force Report. Value Health. 2018;21(2):124-30.

15. Jena A, Lakdawalla D. Value frameworks for rare diseases: should they be different? April 12, 2017. Accessed March 4, 2021. https://www.healthaffairs.org/ do/10.1377/hblog20170412.059563/full/

16. Strouse JJ, Lobner K, Lanzkron S, Haywood Jr C. NIH and National Foundation expenditures for sickle cell disease and cystic fibrosis are associated with PubMed publications and FDA approvals. Blood. 2013;122(21):1739.

17. Neumann PJ, Kim DD, Trikalinos TA, et al. Future directions for cost-effectiveness analyses in health and medicine. Med Decision Making. 2018;38(7):767-77.

18. Jansen J, Incerti $D$, Linthicum $M$. Developing open-source models for the US health system: practical experiences and challenges to date with the opensource value project. Pharmacoeconomics. 2019;37(11):1313-20.

19. Bloom D, Brenzel L, Cadarette D, Sullivan J. Moving beyond traditional valuation of vaccination: needs and opportunities. Vaccine. 2017;35(Suppl 1): A29-35.

20. Bloom D, Canning D, Weston M. The value of vaccination. World Econ. 2005;6(3):15. 